\title{
Direct observation of free-exciton thermalization in quantum-well structures
}

Umlauff, M.; Hoffmann, J.; Kalt, H.; Langbein, Wolfgang Werner; Hvam, Jørn Märcher; Scholl, M.; Söllner, J.; Heuken, M.; Jobst, B.; Hommel, D.

Published in:

Physical Review B

Link to article, DOI:

10.1103/PhysRevB.57.1390

Publication date:

1998

Document Version

Publisher's PDF, also known as Version of record

Link back to DTU Orbit

Citation (APA):

Umlauff, M., Hoffmann, J., Kalt, H., Langbein, W. W., Hvam, J. M., Scholl, M., Söllner, J., Heuken, M., Jobst, B., \& Hommel, D. (1998). Direct observation of free-exciton thermalization in quantum-well structures. Physical Review B, 57(3), 1390-1393. https://doi.org/10.1103/PhysRevB.57.1390

\section{General rights}

Copyright and moral rights for the publications made accessible in the public portal are retained by the authors and/or other copyright owners and it is a condition of accessing publications that users recognise and abide by the legal requirements associated with these rights.

- Users may download and print one copy of any publication from the public portal for the purpose of private study or research.

- You may not further distribute the material or use it for any profit-making activity or commercial gain

- You may freely distribute the URL identifying the publication in the public portal 


\title{
Direct observation of free-exciton thermalization in quantum-well structures
}

\author{
M. Umlauff, J. Hoffmann, and H. Kalt \\ Institut für Angewandte Physik, Universität Karlsruhe, D-76128 Karlsruhe, Germany \\ W. Langbein and J. M. Hvam \\ Mikroelektronik Centret, Technical University of Denmark, DK-2800 Lyngby, Denmark \\ M. Scholl, J. Söllner, and M. Heuken \\ Institut für Halbleitertechnik, RWTH Aachen, D-52074 Aachen, Germany \\ B. Jobst and D. Hommel \\ Institut für Festkörperphysik, Universität Bremen, D-28334 Bremen, Germany
}

(Received 23 September 1997)

\begin{abstract}
We report on a direct observation of free-exciton thermalization in quantum-well structures. A narrow energy distribution of free $1 s$ excitons is created in ZnSe-based quantum wells by emission of one LO phonon after optical excitation of the continuum states with picosecond laser pulses. The subsequent relaxation dynamics within the $1 s$-exciton dispersion is directly monitored by time-resolved studies of the phonon-assisted photoluminescence. It is demonstrated that the free-exciton distribution remains nonthermal for some $100 \mathrm{ps}$. The observed dynamics is in reasonable agreement with numerical results of a rate-equation model which accounts for the relevant exciton-phonon coupling mechanisms. [S0163-1829(98)04703-1]
\end{abstract}

Optical excitation above the band gap of semiconductor quantum structures leads to the creation of electron-hole pairs. These relax in energy and at not too high densities, and lattice temperatures $T_{L}$ eventually form excitons. The explicit path of this relaxation is determined by the strength of the interband Coulomb interaction and the coupling to lattice vibrations. In quantum-well structures of III-V compounds, the electrons and holes are assumed to relax individually toward their respective band extrema, and bind to excitons on a time scale of $10 \mathrm{ps}^{1-4}$ On the other hand, excitons form, directly assisted by emission of optical phonons, which is the predominant mechanism in the more polar II-VI quantum structures. $^{5-7}$

Excitons initially formed from electron-hole pairs usually still have a large kinetic energy compared to $k T_{L}$. At low exciton densities, the thermalization and cooling of these socalled "hot" excitons to states with a small in-plane wave vector $\mathbf{K}_{\|}$is dominated by their interaction with phonons. In quantum wells, the relaxation of free excitons is accompanied by the trapping to local potential minima. This trapping occurs on a subpicosecond time scale, and leads to a spectrally narrow, nonthermal distribution of localized excitons when the emission of one or several optical phonons leads to the region of the localization tail in the excitonic density of states. $^{6-8}$ The dynamics within the localized states is mainly determined by the probability for the excitons of finding an energetically lower minimum within their lifetime. ${ }^{8}$ Depending on the time constants for the various processes described above, the energy distribution even of the free excitons can be far from thermal equilibrium, not only in a short-laserpulse experiment but also under cw excitation. ${ }^{5}$ We will focus in this paper on the relaxation of free nonthermal excitons in quantum wells.
There have been many experimental reports on the relaxation dynamics of quasi-two-dimensional (2D) excitons in semiconductor quantum wells during the recent years. ${ }^{2,6-15}$ But these results are based on time-resolved studies of the zero-phonon line (ZPL). The population of free-exciton states with large $\mathbf{K}_{\|}$is not directly accessible in such experiments, because only excitons with $\mathbf{K}_{\|} \approx 0$ can contribute to the photoluminescence (PL) by direct emission of a photon. Therefore, the relaxation dynamics of hot free excitons can only be investigated indirectly by the PL rise time. In quantum wells, the localized excitons actually give the main contribution to the ZPL. The ZPL rise time is of course fast if the excitation is resonant to the exciton absorption ${ }^{13}$ or if the emission of optical phonons leads to trapping to localized states. $^{6-8,14}$ Slow rise times were found in both III- ${ }^{2,11,12}$ and II-VI quantum wells ${ }^{15}$ under nonresonant excitation. But these experiments cannot distinguish the processes of exciton formation, relaxation by acoustic-phonon emission toward the states at $\mathbf{K}_{\|} \approx 0$, and the trapping and migration in local potential minima. So it is not surprising that these studies lead to contradictory results, e.g., for the exciton formation time, ${ }^{2,11}$ and that the measured excitonic energy loss rate lies one order of magnitude below the theoretically expected value for free quasi-2D excitons. ${ }^{12,16}$

However, the population dynamics of the hot free exciton states can be visualized directly in the energy-time coordinates by time-resolved studies of the LO-phonon-assisted PL. The matrix element for the LO-phonon-assisted recombination process can be considered to be independent of $\mathbf{K}_{\|},{ }^{17}$ and therefore the PL intensity at the photon energy $E_{\mathrm{ME}}+E\left(\mathbf{K}_{\|}\right)-E_{\mathrm{LO}}$ monitors the population of exciton states with kinetic energy $E\left(\mathbf{K}_{\|}\right)$(see Fig. 1). $E_{\mathrm{ME}}$ denotes the energy of the mobility edge, separating free and localized exciton states, and $E_{\mathrm{LO}}$ is the LO-phonon energy $(31 \mathrm{meV}$ in 


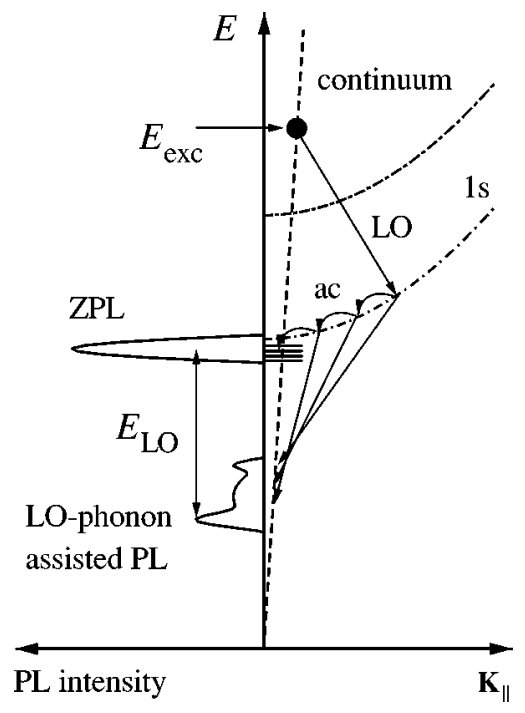

FIG. 1. Schematic drawing of the exciton relaxation by LOphonon (LO) and acoustic-phonon (ac) emission and of the resulting zero-phonon line (ZPL) and LO-phonon-assisted PL. The dashed line is the photon dispersion, and the dash-dotted lines represent the dipersion of the $1 s-\mathrm{HH}$ excitons and the onset of the exciton continuum. The horizontal lines indicate the localized exciton states. Light-hole states are omitted for simplicity.

$\mathrm{ZnSe}$ ). This method has been used successfully to investigate the thermalization dynamics of free excitons in $\mathrm{Cu}_{2} \mathrm{O}$ bulk material. $^{18}$

In this paper we report on a direct observation of the thermalization dynamics of free excitons in quantum-well structures. ZnSe-based quantum wells are well suited for such kinds of studies for two reasons. First, the LO-phonon sideband in $\mathrm{ZnSe}$ is relatively bright, due to the strong Fröhlich coupling in this polar semiconductor (but still about two orders of magnitude less intense compared to the ZPL). Second: The quality of the available samples has improved, i.e., the defect density has been reduced, and the inhomogeneous width of the ZPL band has become several times smaller than $E_{\mathrm{LO}}$. It is important to make sure that no other luminescence, which could superimpose the hot-exciton features, appears in the vicinity of the LO-phonon replica.

We have obtained similar results for different $\mathrm{ZnSe}$ quantum-well samples with ternary ZnSSe as well as with quaternary $\mathrm{ZnMgSSe}$ barriers, grown by metal-organic vapor-phase epitaxy (MOVPE) and molecular beam epitaxy (MBE). In this short paper we restrict ourselves to a study of a 120-period $\mathrm{ZnSe}_{\mathrm{ZnS}} \mathrm{Zn}_{0.1} \mathrm{Se}_{0.9}$ superlattice grown by MOVPE on a (001) GaAs substrate, the sample which reveals the hot exciton relaxation dynamics the most evidently. The width of the wells and of the barriers are 7 and $11 \mathrm{~nm}$, respectively. Apart from the phonon replica, the timeintegrated PL spectrum of the sample for nonresonant excitation, i.e., at a photon energy far above the band gap of the barrier material, consists of three lines labeled HH (heavy hole), LH (light hole), and BE (bound exciton) in Fig. 2. The full width at half maximum of these lines is $4 \mathrm{meV}$, indicating the inhomogeneous broadening of the exciton resonances. Because the energy positions of the lines $\mathrm{HH}$ and $\mathrm{LH}$ $\left(E_{\mathrm{HH}}=2.816 \mathrm{eV}\right.$ and $\left.E_{\mathrm{LH}}=2.829 \mathrm{eV}\right)$ correspond to distinct resonances in the absorption spectrum as well as in the

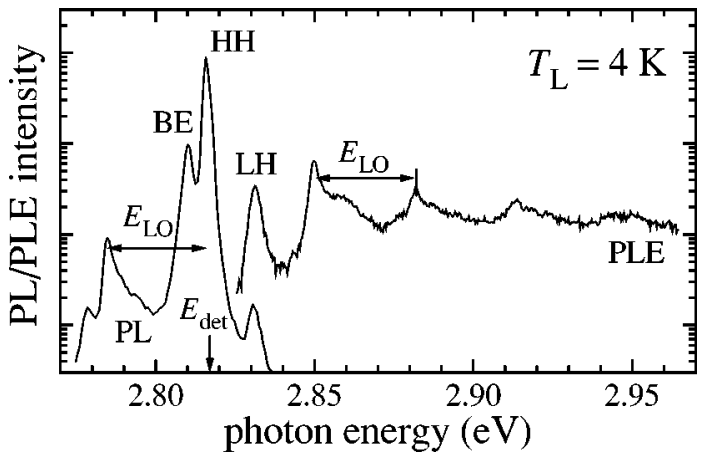

FIG. 2. Time-integrated PL spectrum of the ZnSe/ZnSSe SL sample for nonresonant excitation and PLE spectrum for a detection photon energy $E_{\text {det }}$ within the $\mathrm{HH}$ exciton line.

reflexion spectrum (not displayed here), we can attribute them to the recombination of $\mathrm{HH}$ and $\mathrm{LH}$ excitons. We want to point out that no luminescence band appears between the energy position of the $\mathrm{BE}$ line and $E_{\mathrm{HH}}-E_{\mathrm{LO}}$ under those nonresonant excitation conditions. The PL intensity in that spectral region is almost ten times lower compared to the LO-phonon replica of the HH line.

The photoluminescence excitation (PLE) spectra of the sample (Fig. 2) show a pronounced phonon cascade, ${ }^{19}$ reflecting the strong exciton-LO-phonon coupling. The fact, that the energy separation $\Delta E$ of the PLE maxima corresponds exactly to $E_{\mathrm{LO}}$ gives clear evidence that the relaxation toward the radiative states is governed by excitons and not by free carriers.

Figure 3 shows the PL spectra of the sample for $\mathrm{cw}$ excitation at different photon energies $E_{\text {exc }}$. For clarity, the baselines have been shifted. $E_{\text {exc }}$ varies between $E_{\mathrm{HH}}+1.2 E_{\mathrm{LO}}$ (curve $a$ ) and $E_{\mathrm{HH}}+1.5 E_{\mathrm{LO}}$ (curve $d$ ). As described in Fig. 1 , for such an excitation energy, an exciton in the $1 s-\mathrm{HH}$ state can be formed rather quickly from the continuum states by emission of one LO phonon. This scattering process is expected to happen within at most a few picoseconds. ${ }^{7}$ The

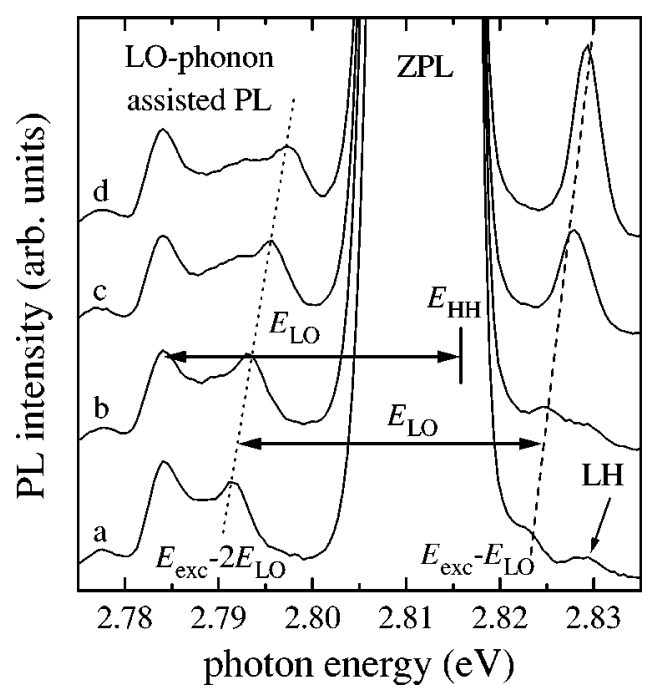

FIG. 3. PL spectra of the sample for $\mathrm{cw}$ excitation with excess energies between 1.2 $E_{\mathrm{LO}}$ (curve $a$ ) and 1.5 $E_{\mathrm{LO}}$ (curve $d$ ) with respect to the maximum of the $\mathrm{HH}$ line. The dashed (dotted) line corresponds to $E_{\mathrm{exc}}-E_{\mathrm{LO}}\left(E_{\mathrm{exc}}-2 E_{\mathrm{LO}}\right)$. 
remaining kinetic energy $E\left(\mathbf{K}_{\|}\right)$of the $1 s$ exciton can then only be dissipated by a cascade of interactions with acoustic phonons-because of the lack of final exciton states for further LO-phonon scattering. In contrast to the LO-phonon coupling, there exists no fixed energy transfer for the relaxation of excitons by emission of acoustic phonons, so that at low lattice temperatures all exciton states between $E_{\text {exc }}$ $-E_{\mathrm{LO}}$ and $E_{\mathrm{HH}}$ can act as intermediate states for the relaxation process, and will be occupied to some extent. Because of the $\mathbf{K}$-selection rule, the direct hot-exciton luminescence at energy $E_{\mathrm{exc}}-E_{\mathrm{LO}}$ is very weak. This emission is enhanced when after the emission of the first LO phonon the LHexciton resonance is reached. The LH excitons at $\mathbf{K}_{\|} \approx 0$ can recombine without emission of an additional further phonon (see Fig. 3). In contrast to that, the hot-exciton feature in the LO-phonon-assisted PL $\left(E_{\mathrm{exc}}-2 E_{\mathrm{LO}}\right)$ and the LO-phonon replica of the $\mathrm{HH}$ line $\left(E_{\mathrm{HH}}-E_{\mathrm{LO}}\right)$ are of almost equal intensity. The line shape of the phonon-assisted PL reflects the population of the 1s-HH exciton states, but not the density of states at these photon energies. Especially, it does not reflect the population of localized hh-exciton states, because the density of localized states is negligible in this spectral region.

Within the LO sideband, the hot-exciton peak is accompanied by a luminescence band at the low-energy tail, which reflects the ongoing exciton relaxation by acoustic-phonon emission. This relaxation dynamics of the free excitons is directly monitored in time-resolved PL studies of the sideband. We excited the sample with the ps pulses of a frequency-doubled titanium-sapphire laser at a repetition rate of $76 \mathrm{MHz}$. The pulse energy was reduced to $4 \mathrm{pJ}$, to avoid exciton-exciton scattering as far as possible. The initial electron-hole pair density per quantum well was estimated to $10^{9} \mathrm{~cm}^{-2}$. The PL was recorded using a spectrometer, a synchroscan streak-camera and a charge coupled device array, giving a time resolution of $30 \mathrm{ps}$. The PL spectra recorded at different times after the excitation pulse directly visualize the relaxation dynamics of the free quasi-2D excitons. They are snapshots of the exciton population at the respective time.

Due to the fast LO-phonon scattering process onto the $1 s-\mathrm{HH}$ exciton dispersion, which is beyond the time resolution of our experiment, the hot-exciton peak develops simultaneously with the laser pulse (Fig. 4). The created $1 s$ excitons have an initial kinetic energy with respect to the minimum of their dispersion curve which is determined by the photon energy of the laser $E_{\text {exc }}$. Examples for excess energies of 8 and $15 \mathrm{meV}$, respectively, are shown in Fig. 4. The narrow nonthermal exciton distribution develops toward a thermal distribution by emission of acoustic phonons via the piezoelectric and deformation potential coupling. After 50 ps the PL spectrum is essentially flat, i.e., the excitons are spread over all states below $E_{\mathrm{exc}}-E_{\mathrm{LO}}$. With progressing time, the population of exciton states with high kinetic energy is further reduced and the HH line develops. The emission peak at $\mathrm{E}_{\mathrm{hh}}-\mathrm{E}_{\mathrm{LO}}$ has exactly the same temporal evolution as the ZPL. One can easily see that the rise is slow, and that the maximum of this emission occurs later than $100 \mathrm{ps}$ after excitation. Within the same time the high-energy tail of the exciton distribution becomes exponential, i.e., it can be described by a Boltzmann distribution function, as expected

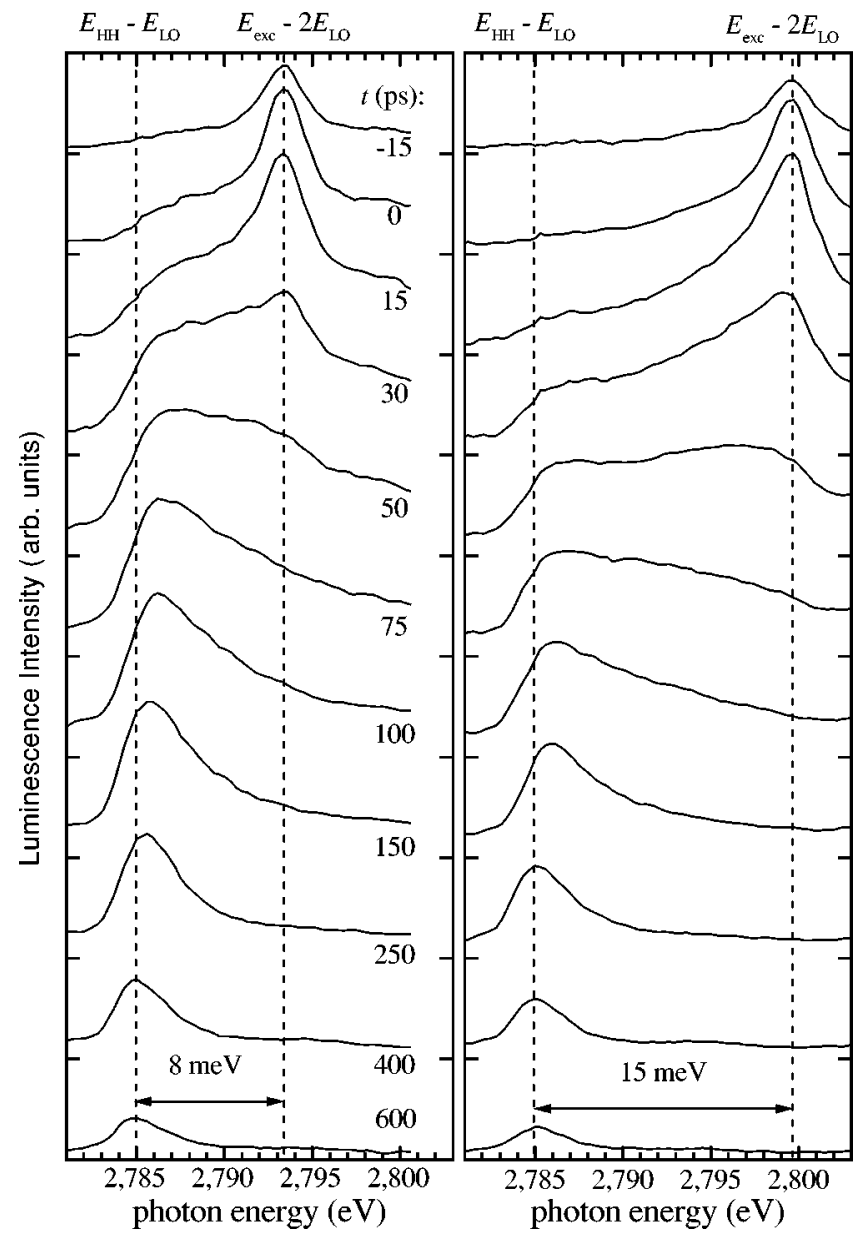

FIG. 4. LO-phonon-assisted PL of the sample at $4 \mathrm{~K}$ for different times $t$ after the excitation pulse (baselines are shifted). The photon energy of the excitation laser $E_{\mathrm{exc}}$ is $E_{\mathrm{HH}}+E_{\mathrm{LO}}+8 \mathrm{meV}$ in (a), and $E_{\mathrm{HH}}+E_{\mathrm{LO}}+15 \mathrm{meV}$ in (b).

for thermal quasiequilibrium. Then, the temperature of the exciton distribution cools down toward the temperature of the phonon system $T_{\mathrm{L}}=4 \mathrm{~K}$. Finally, the $\mathrm{HH}$ line decays with a time constant of $\approx 200$ ps by exciton recombination. We can estimate the energy-loss rate of the hot quasi-2D excitons from the change of the average exciton energy as a function of time. The value depends on the initial kinetic energy of the excitons and varies between -0.033 and $-0.08 \mathrm{meV} / \mathrm{ps}$.

Photon-emission processes after relaxation assisted by optical phonons are often discussed in terms of hot luminescence or coherent Raman scattering. ${ }^{6,7}$ A priori, these two processes are indistinguishable within the dephasing time of the emitting state, which cannot be deduced from our experiments. But it is evident from the temporal evolution of the spectra in Fig. 4 that the dynamics of a real population of excitons is monitored: the spectrally integrated luminescence transients (not shown) reveal a single exponential decay (the decay time is approximately equal to $200 \mathrm{ps}$ ) with no indication for a fast additional contribution right after the laser pulse. The latter would be expected if the signal at $\mathrm{E}_{\text {exc }}$ $-2 E_{\mathrm{LO}}$ were dominated by a coherent emission decaying with the excitonic phase relaxation time of typically several picoseconds.

The observed dynamics is in qualitative agreement with 


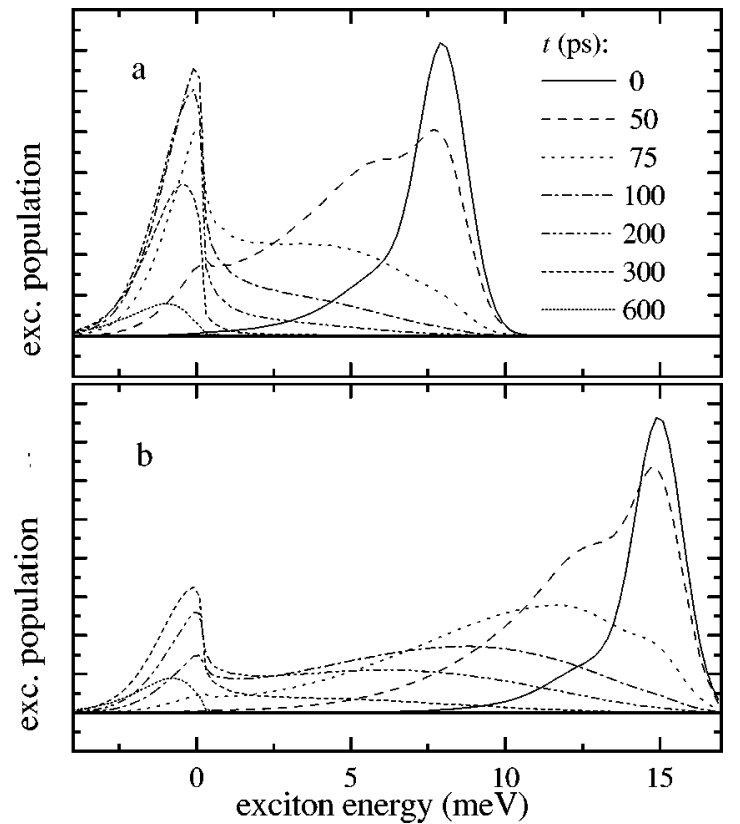

FIG. 5. Computed exciton distribution function at different times $t$ after short pulse excitation for the same excess energies as in Fig. 4. The origin of the energy scale corresponds to $E_{\mathrm{ME}}$.

theoretical predictions for the relaxation of free excitons due to acoustic phonon emission. Figure 5 shows the numerical results for the dynamical evolution of the exciton distribution based on a rate-equation model which accounts explicitly for deformation potential and piezo-electric coupling and coupling to localized tail states. ${ }^{20} \mathrm{We}$ have computed the exciton-phonon scattering rates with Fermi's golden rule, assuming a quasi-2D envelope function for the excitons. ${ }^{16}$

A quantitative comparison between experiment and simulation yields that the measured relaxation is faster by a factor of 2. This difference is not due to exciton-exciton scattering: the dynamics is not density dependent at all below $3 \times 10^{8}$ $\mathrm{cm}^{-2}$, and only slightly modified for the data in Fig. 4. Besides this difference, the reason of which is subject of further investigations, all significant features of the experiment are reproduced. In a previous report on exciton relaxation in GaAs quantum wells, ${ }^{12}$ a surprisingly slow exciton relaxation was deduced from time-resolved studies of the ZPL. But the ZPL is always affected by the dynamics within the localized tail states.

To summarize, we have shown that in $\mathrm{ZnSe}$-based quantum structures the relaxation of photocreated electron-hole pairs to the radiative states is governed by the $1 \mathrm{~s}$ excitons. We visualized the relaxation dynamics of free quasi-2D excitons to the states with $\mathbf{K}_{\|} \approx 0$ using the technique of timeresolved PL studies of the LO-phonon sideband. The thermalization of hot excitons created with an initial kinetic energy of about half a LO-phonon energy has been demonstrated to happen within some hundred picoseconds. The relaxation dynamics is in qualitative agreement with the theoretical prediction for acoustic-phonon scattering.

The authors acknowledge helpful discussions with R. von Baltz (University Karlsruhe). This work was supported by the Deutsche Forschungsgemeinschaft.
${ }^{1}$ J. Shah, Ultrafast Spectroscopy of Semiconductors and Semiconductor Nanostructures, Springer Series in Solid-State Sciences, Vol. 115 (Springer, Berlin, 1996).

${ }^{2}$ T. C. Damen et al., Phys. Rev. B 42, 7434 (1990).

${ }^{3}$ P. W. M. Blom et al., Phys. Rev. Lett. 71, 3878 (1993).

${ }^{4}$ R. Kumar et al., Phys. Rev. B 54, 4891 (1996).

${ }^{5}$ S. Permogorov, Phys. Status Solidi B 68, 9 (1975).

${ }^{6}$ R. P. Stanley et al., Phys. Rev. Lett. 67, 128 (1991).

${ }^{7}$ N. Pelekanos et al., Phys. Rev. B 43, 9354 (1991).

${ }^{8}$ H. Kalt et al., Phys. Rev. B 45, 4253 (1992).

${ }^{9}$ F. Yang et al., Phys. Rev. B 53, R1697 (1996).

${ }^{10}$ Z. L. Yuan et al., J. Appl. Phys. 79, 424 (1996).

${ }^{11}$ J. Kusano et al., Phys. Rev. B 40, 1685 (1989).

${ }^{12}$ Y. Masumoto, S. Shionoya, and H. Kawaguchi, Phys. Rev. B 29, 2324 (1984).

${ }^{13}$ A. Schülzgen et al., J. Cryst. Growth 138, 575 (1994); U. Neu- kirch et al., ibid. 138, 849 (1994).

${ }^{14}$ U. Neukirch et al., J. Cryst. Growth 138, 861 (1994).

${ }^{15}$ R. Hellmann et al., J. Cryst. Growth 138, 791 (1994); M. Godlewski et al., ibid. 159, 533 (1996).

${ }^{16}$ T. Takagahara, Phys. Rev. B 31, 6552 (1985).

${ }^{17} \mathrm{~S}$. Permogorov, in Excitons, edited by E. I. Rashba and M. D. Sturge (North-Holland, Amsterdam, 1982).

${ }^{18}$ D. W. Snoke, D. Braun, and M. Carnoda, Phys. Rev. B 44, 2991 (1991).

${ }^{19}$ R. P. Stanley and J. Hegarty, in Optics of Semiconductor Nanostructures, edited by F. Henneberger, S. Schmitt-Rink, and E. O. Göbel (Akademie Verlag, Berlin, 1993).

${ }^{20}$ For the deformation potentials we used $D_{c}=-5.9 \mathrm{eV}$ and $D_{v}=$ -1.0 eV. G. Schötz, Ph.D. thesis, Universität Regensburg, 1995). The piezoelectric coefficient is $e_{14}=0.049 \mathrm{C} / \mathrm{m}^{2}$. 